\title{
Development of protective antibodies to Streptococcus pneumoniae in healthy children
}

\author{
David Björnheden $^{1}$, Bertil Kaijser ${ }^{2}$, Bill Hesselmar $^{{ }^{*}}$ \\ ${ }^{1}$ Institute for Clinical Science, Department of Paediatrics, The Sahlgrenska Academy, University of Gothenburg, Gothenburg, Sweden \\ ${ }^{2}$ Department of Clinical Bacteriology, The Sahlgrenska Academy, University of Gothenburg, Gothenburg, Sweden \\ Email: ${ }^{\text {bill.hesselmar@vgregion.se }}$
}

Received 10 June 2011; revised 22 September 2011; accepted 20 October 2011

\begin{abstract}
Aim: To study how immunity to Streptococcus pneumoniae normally develops in healthy children. Methods: Ninety two healthy children at 3-5, 7 - 9 and 13 15 years of age were recruited. No one of the children had previously been given pneumococcal vaccine. Serum was analysed for pooled antigens of the 23 most common pneumococcal polysaccharides with ELISA technique, and results are given in opitical density (OD). A three-level semi-quantitative system was used to assess degree of immunity to Streptococcus pneumoniae. Cut-off levels were $\mathrm{OD} \leq 0.7$ and $\mathrm{OD}$ $\geq 1.3$, separating low, intermediate and high degree of immunity. Results: Median values for OD differed significantly between the groups, with OD $0.91,1.18$ and 1.10 respectively $(p=\mathbf{0 . 0 0 4})$. Levels were lower in the youngest age group, but from age 7 - 9 years, levels were similar trough out childhood. Twenty six percent of the children in the youngest age-group had a low degree of protection (OD $\leq 0.7)$ to Streptococcus pneumoniae. Such low levels were uncommon from 7 - 9 years of age, found in only $13 \%$ of the children. Conclusion: Protective antibodies to Streptococcus pneumoniae develops mainly during the preschool period. Thereafter, levels are stable throughout childhood up to the age of 15 years.
\end{abstract}

Keywords: Antibody Formation; Child; Streptococcus Pneumoniae

\section{INTRODUCTION}

Infections are common in children, especially in the younger age groups. With increasing age, however, infections become less frequent. This decline in infectious burden is well illuminated by the number of antibiotic prescriptions per year in children (Figure 1). In Sweden, phenoxymethylpenicillin $(\mathrm{PcV})$ is prescribed three times

\footnotetext{
${ }^{*}$ Corresponding author.
}

as often in 3 - 5 year olds as compared to teenagers, and amoxicillin eight times as often. Infections due to Streptococcus pneumoniae are common in both children and adults, and pneumoсоссi are the cause in about one third of all community acquired pneumonias in children [1]. Pneumococci is also commonly found in otitis media in children [2], and high mortality rates are associated with infectious meningitis caused by pneumococci [3].

Recurrent infections are common in younger childhood, but it is not always easy to know in a particular child if the frequency and severity of infections should be considered as abnormal, or if it is within the "normal" variation. The susceptibility to become infected shows a great inter-individual variation, and not seldom are recurrent infections a result of increased exposure to infectious agents at day care etc during a time period when the immune system is immature [4]. In recurrent systemic pneumococcal infections, however, an underlying disease is often the cause [5]. These underlying diseases are not necessarily related to immune deficiency, other common chronic diseases and conditions in children may also increase the risk for infections with bacteria as Streptococcus pneumoniae [6]. But an increased susceptibility to infections, and infectious-related lung damages, could also be due to a disability to produce antibodies to pneumococci and other capsulated bacteria [7].

In the evaluation of children with recurrent infections, bronchiectasis, chronic wet cough and purulent bronchitis, the laboratory work often includes the measurement of antibodies to Streptococcus pneumoniae, and the response to pneumococcal vaccine. But the interpretations of these antibody tests are often difficult, since there are limited reference values for the normal development of immunity to pneumococci in children. It has been studied up to the age of 2 years [8], but the time course for the normal development of protective antibodies to Streptococcus pneumoniae throughout childhood is less well studied. The aim of the present study was to analyse the pattern and normal development of antibodies to Streptococcus pneumoniae in children at different ages. 


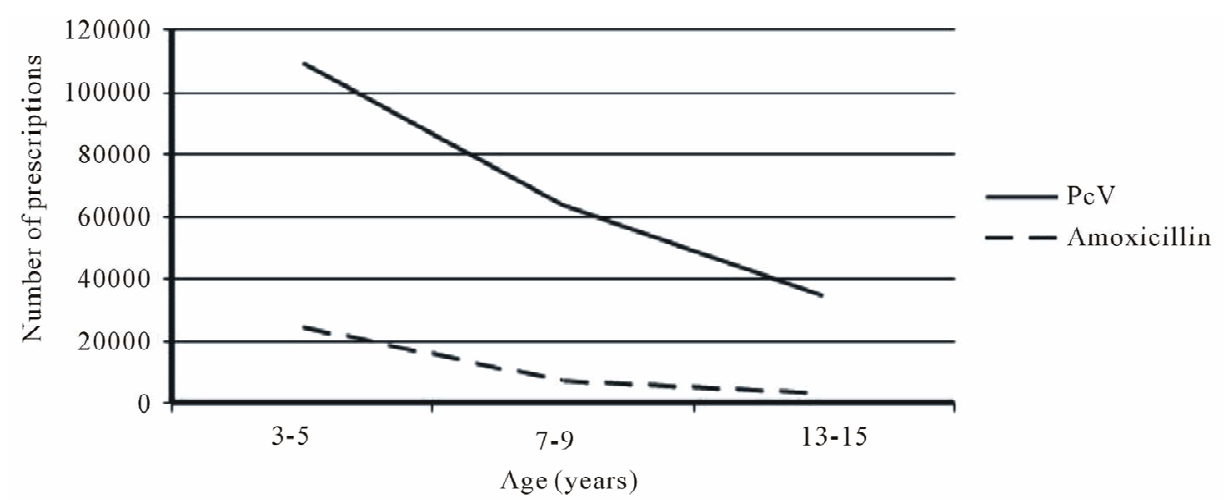

Figure 1. The number of $\mathrm{PcV}$ and amoxicillin prescriptions to children in Sweden in 2008. Data from Apoteket, The Swedish Pharmacy Agency.

\section{METHOD}

Ninety-two healthy children were included in the study. They were divided into three groups; 3 - $5(\mathrm{n}=31), 7$ - 9 $(\mathrm{n}=31)$ and $13-15(\mathrm{n}=30)$ years of age with an equal gender distribution. Recruited children were all to have minor surgery, and blood samples for antibody analysis were collected at time for surgery. Before entering the study, parents and patients were interviewed regarding concomitant diseases. Children were excluded if they were born preterm, if they had any chronic disease, if they had any disease or condition that have required frequent hospital visits, if they had a history of allergic symptoms, if they were prone to infections (e.g. $>2$ reported otitis media, ear drainage tubes, $>1$ reported pneumonia or a history of any other severe infection), or if they had an ongoing infection. No child had received pneumococcal vaccination; the blood samples for the study were collected during 2004 - 2006, i.e. 3 - 5 year before pneumococcal vaccine was included in the regular vaccination program.

The antibody determination was performed using standard ELISA (Enzymelinked Immuno Assay) procedures. The vaccine Pneumovax ${ }^{\circledR}$ was used as antigen. The Pneumovax contains 25 microgram each of the 23 most common pneumococcal capsule polysacharide antigens. The results are given in Optical Density (OD).

A semi-quantitative system, based on an adult reference population, was used to score the degree of immunity to Streptococcus pneumoniae. Levels are: "low titer" $(\mathrm{OD} \leq 0.7)$ with low or no immunity to Streptococcus pneumoniae. "Medium titers" $(0.7<\mathrm{OD}<1.3)$ with limited immunity, and "high titer $(\mathrm{OD} \geq 1.3)$ with probable immunity to pneumococci infections.

Kruskal-Wallis test was used to test for differences between groups.

The study was approved by The Ethics Committee at The Sahlgrenska Academy, University of Gothenburg, Sweden. A Consent Form was signed by the parents/ guardians, and by the children/teenagers if they were old enough.

\section{RESULTS}

Median values for optical density for each age group are shown in Figure 2. A significant difference was seen between the age groups, with lower levels in the youngest children.

From age 7-years, however, levels were similar throughout childhood.

Based on the semi-quantitative system for interpreting degree of immunity, only $6 \%$ in the youngest age-group had acquired a high degree of protection against Streptococcus pneumoniae. $68 \%$ had intermediate and $26 \%$ low degree of immunity (Figure 3). The corresponding figures for 7 - 9 and 13 - 15 year olds were $(26 \%, 61 \%$ and $13 \%)$ and $(23 \%, 63 \%$ and $13 \%)$ respectively.

\section{DISCUSSION}

The aim of the study was to investigate to what degree healthy children have developed protective antibodies to a pooled sample of the 23 most common Streptococcus pneumoniae polysaccharide antigens, and how this immunity develops over time. We found that children between 3 - 5 years of age have low levels, i.e. a lesser degree of acquired immunity to pneumococci than older children. From 7 years of age, the degree of immunity is stable throughout childhood and adolescence up to age 15 years. The lesser degree of immunity in younger children is probably a consequence of exposure and maturation; it takes a few years before the child has been exposed to different pneumococci infections, severe enough to stimulate an immune response, and the immune system has to be mature enough to respond to these types of capsulated bacteria. Infants and young children, with an immature immune system, have difficulties in recognizing polysaccharide antigens from capsulated bacteria [9]. The most remarkable finding from the study was, how- 


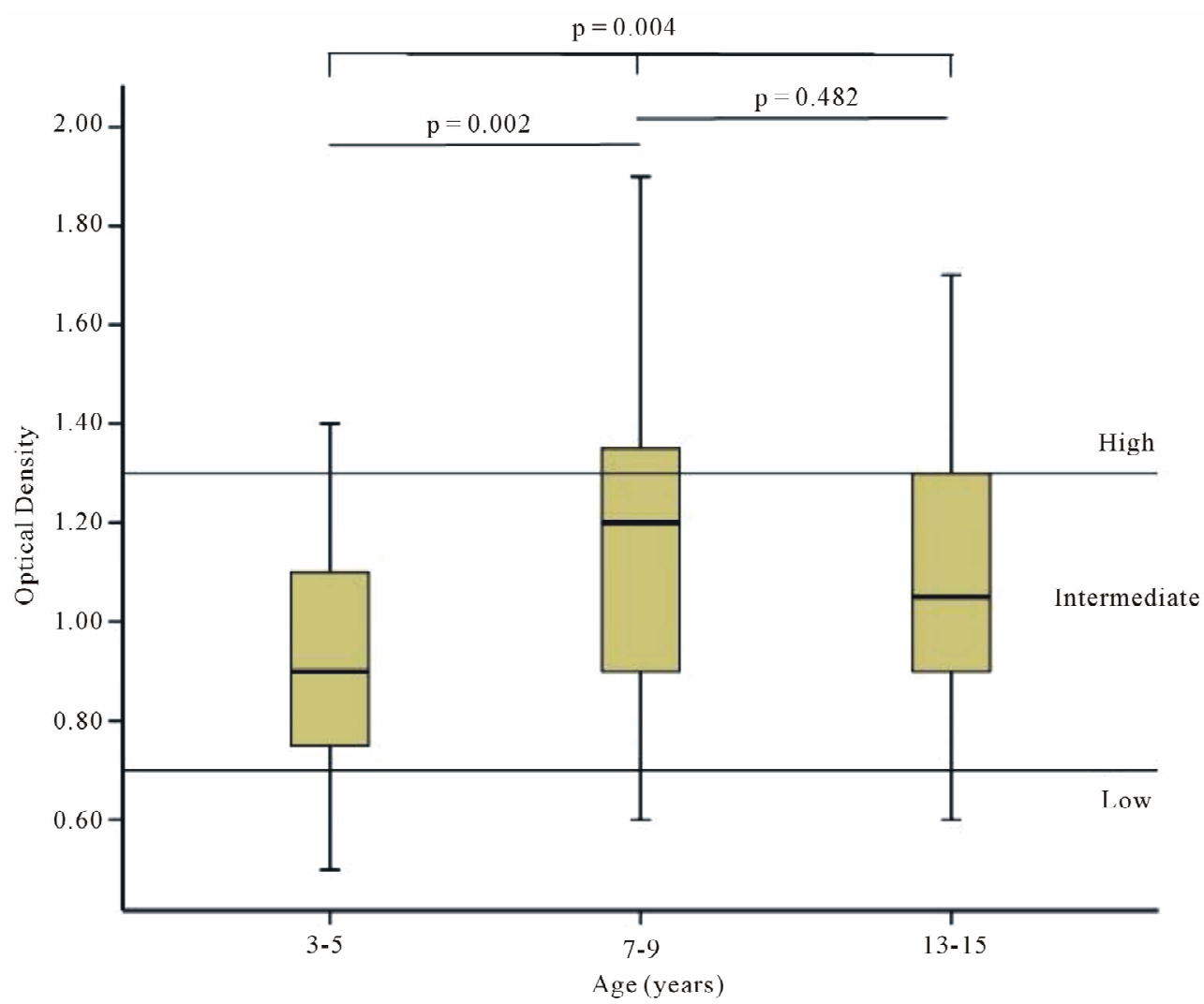

Figure 2. Boxes show median values, 25th and 75th percentiles (Tukey's Hinges), and error bars 95\% CI for optical density (OD) in the three different age groups. Reference lines represent "low" and "high" degree of immunity.

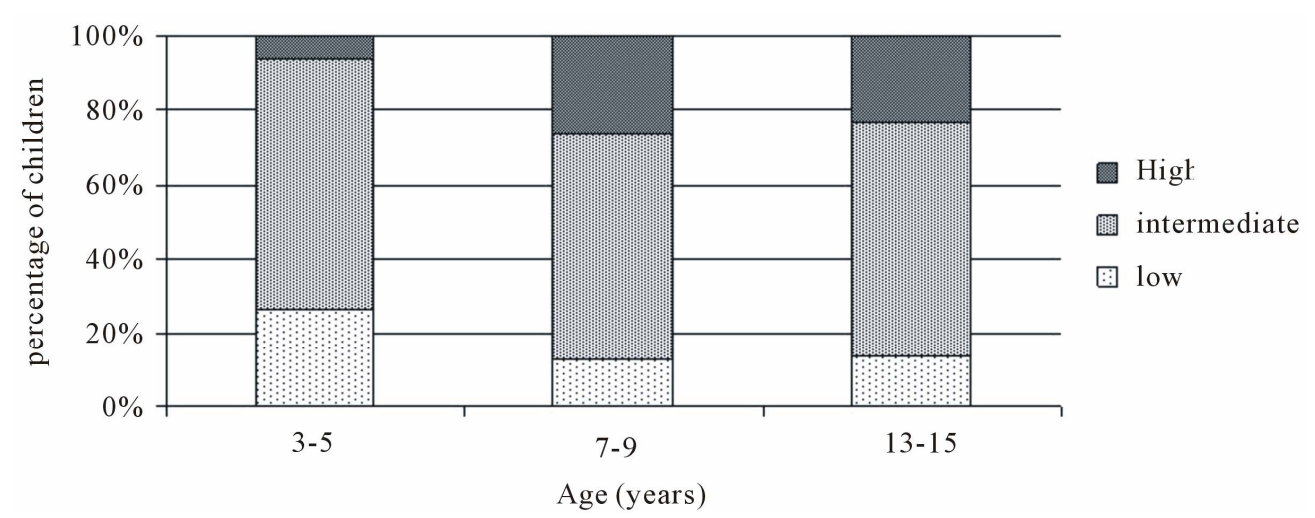

Figure 3. Degree of immunity to Streptococcus pneumoniae in three different age groups. Low: optical density $(\mathrm{OD}) \leq 0.7$, medium: $0.7<\mathrm{OD}<1.3$, high: $\mathrm{OD} \geq 1.3$.

ever, that the plateau in antibody levels was reached already at 7 - 9 years of age. This finding does not necessarily mean that immunity to pneumococci isn't broadened to include more serotypes with increasing age, but it indicate that more than $80 \%$ of healthy children from 7 years of age have some level of immune response to Streptococcus pneumoniae.

The intension with the study was to study normal development of acquired immunity to pneumococci over time in children with a method often used in the immunological framework of analyses when examining children prone to recurrent or severe infections. This ability to develop antibodies to pneumococci, either after infections or after vaccinations, is probably measured with different methods and techniques at different laboratories. We chose a method covering the same 23 polysaccharide antigens as in the 23-valent unconjugated pneumococcal vaccine, since these serotypes reflect the most common 
pneumococci affecting children. They are also the set of combined serotypes often measured when evaluating response to pneumococcal vaccination. Even though this method is a standard procedure at several hospital laboratories, the interpretation of the test is often difficult when it comes to children since "reference values" are lacking. The present data could therefore give valuable information for the clinician in interpreting such test results; a low titer to pneumococci is not uncommon in unvaccinated pre-school children. From age 7, however, a low antibody titer to pneumococci is uncommon, present in only 13 percent of the children. Even though not specifically investigated in this study, one may presume that the general findings from the study could be extrapolated to include also other methods used to measure antibodies to pneumococci; i.e. a low antibody titer is not uncommon in preschool children, but is seldom seen from age 7 years.

The semi-quantitative method with arbitrary cut-off levels, used to assess immunity to pneumococcal infections in adults, may be used as a rough instrument to estimate immunity also in children. One may assume that a high level $(O D \geq 1.3$ ) would stand for immunity in children as well as in adults. The majority of children had intermediate $(0.7<\mathrm{OD}<1.3)$ levels, and these levels probably reflect a normal immune response in relation to "normal exposure". The question is, however, more complex when it comes to low levels ( $\mathrm{OD} \leq 0.7)$. The children in the study were not prone to infections, and none had received pneumococcal vaccine. One may therefore assume that these low levels are due to low exposure rates in these particular children, and not immune deficiency or disability to produce antibodies to capsulated bacteria such as Streptococcus pneumoniae. In children with recurrent or severe infections, however, a low antibody level $(\mathrm{OD} \leq 0.7)$ to pneumococci should lead to further immunological evaluations, evaluations that often include measurement of response to pneumococcal vaccination [10]. Even though not tested in the present study, one may assume that children from age 7 years should respond to vaccination with the 23-valent unconjugated pneumococcal vaccine with at least a post-vaccination optical density titer of $>0.7$, since almost all healthy children reach this level on normal exposure. This suggestion is further supported from vaccination studies, since most children from 5 years of age reach protective antibody levels after immunization with the 23-valent unconjugated pneumococcal vaccine [11], and a clinical protection from vaccination with the unconjugated vaccine is seen already from 2 years of age [12].

In conclusion, the present study reflects how immunity to Streptococcus pneumoniae develops over time in children. The data may help clinicians in interpreting im- mune test results from children prone to infections.

\section{ACKNOWLEDGEMENTS}

The study was supported by grants from Stiftelsen Samariten, Sachsska Barnsjukhuset, Södersjukhuset, Stockholm, Sweden.

\section{REFERENCES}

[1] Heiskanen-Kosma, T., Korppi, M., Jokinen, C., Kurki, S., Heiskanen, L., Juvonen, H., et al. (1998) Etiology of childhood pneumonia: Serologic results of a prospective, population-based study. Pediatric Infectious Disease Journal, 17, 986-991. doi:10.1097/00006454-199811000-00004

[2] Coker, T.R., Chan, L.S., Newberry, S.J., Limbos, M.A., Suttorp, M.J., Shekelle, P.G., et al. (2010) Diagnosis, microbial epidemiology, and antibiotic treatment of acute otitis media in children: A systematic review. Journal of the American Medical Association, 304, 2161-2169. doi:10.1001/jama.2010.1651

[3] Berg, S., Trollfors, B., Claesson, B.A., Alestig, K., Gothefors, L., Hugosson, S., et al. (1996) Incidence and prognosis of meningitis due to Haemophilus influenzae, Streptococcus pneumoniae and Neisseria meningitidis in Sweden. Scandinavian Journal of Infectious Diseases, 28, 247-252. doi:10.3109/00365549609027166

[4] De Martino, M. and Ballotti, S. (2007) The child with recurrent respiratory infections: Normal or not? Pediatric Allergy and Immunology, 18, 13-18. doi:10.1111/j.1399-3038.2007.00625.x

[5] Mason, E.O. Jr., Wald, E.R., Tan, T.Q., Schutze, G.E., Bradley, J.S., Barson, W.J., et al. (2007) Recurrent systemic pneumococcal disease in children. Pediatric Infectious Disease Journal, 26, 480-484. doi:10.1097/INF.0b013e31805ce277

[6] Fletcher, M.A., Laufer, D.S., McIntosh, E.D., Cimino, C. and Malinoski, F.J. (2006) Controlling invasive pneumococcal disease: Is vaccination of at-risk groups sufficient? International Journal of Clinical Practice, 60, 450-456. doi:10.1111/j.1368-5031.2006.00858.x

[7] Miravitlles, M., Vendrell, M., de Gracia, J. (2005) Antibody deficiency in bronchiectasis. European Respiratory Journal, 26, 178-180. doi:10.1183/09031936.05.00027605

[8] Rapola, S., Jantti, V., Haikala, R., Syrjanen, R., Carlone, G.M., Sampson, J.S., et al. (2000) Natural development of antibodies to pneumococcal surface protein A, pneumococcal surface adhesin $\mathrm{A}$, and pneumolysin in relation to pneumococcal carriage and acute otitis media. Journal of Infectious Diseases, 182, 1146-1152. doi:10.1086/315822

[9] Klein Klouwenberg, P. and Bont, L. (2008) Neonatal and infantile immune responses to encapsulated bacteria and conjugate vaccines. Clinical and Developmental Immunology, 2008, 628963.

[10] Tuerlinckx, D., Vermeulen, F., Pekus, V., de Bilderling, G., Glupczynski, Y., Collet, S., et al. (2007) Optimal assess- 
ment of the ability of children with recurrent respiratory tract infections to produce anti-polysaccharide antibodies. Clinical and Experimental Immunology, 149, 295-302. doi:10.1111/j.1365-2249.2007.03409.x

[11] Temple, K., Greenwood, B., Inskip, H., Hall, A., Koskela, M. and Leinonen, M. (1991) Antibody response to pneumococcal capsular polysaccharide vaccine in African chil- dren. Pediatric Infectious Disease Journal, 10, 386-390. doi:10.1097/00006454-199105000-00008

[12] Fiore, A.E., Levine, O.S., Elliott, J.A., Facklam, R.R. and Butler, J.C. (1999) Effectiveness of pneumococcal polysaccharide vaccine for preschool-age children with chronic disease. Emerging Infectious Diseases, 5, 828-831. doi:10.3201/eid0506.990616 